\title{
No Higher Limit
}

National Cancer Institute

\section{Source}

National Cancer Institute. No Higher Limit. NCI Thesaurus. Code C154478.

A higher limit of the normal range does not exist. 